\title{
Preface Issue 4-2016
}

\author{
Hans-Christoph Grunau ${ }^{1}$
}

Published online: 20 September 2016

(C) Deutsche Mathematiker-Vereinigung and Springer-Verlag Berlin Heidelberg 2016

In solid state physics, the difference between conductors and insulators is modelled and explained. In conductors, electrons can move freely through the solid. In an insulator, on the other hand, the electrons are forced to stay close to respective atoms of the solid. This phenomenon can be understood in the realm of quantum mechanics by the so-called band model. Insulators are characterised by completely occupied bands and an energy gap to the first unoccupied band. As recently discovered, such a filled band can, moreover, have topological invariants. Such "topological insulators" are the subject of the survey article by Hermann Schulz-Baldes. These novel topological properties are responsible for the existence of supplementary conducting modes on boundaries of the system: A topological insulator becomes an excellent conductor on its surface.

Certainly, a clothesline will move downwards if one hangs something on it. Similarly one may wonder whether a thin clamped elastic plate will move downwards when being pushed downwards. In terms of the modelling differential equation and boundary conditions the question would be whether a positive source will yield a positive solution. As Guido Sweers outlines in his survey "On sign preservation for clotheslines, curtain rods, elastic membranes and thin plates" the answer is in some cases, as expected, positive. But there are also, at a first glance counterintuitive, models where such a sign preservation property may fail. Sometimes, sign preservation and its failure may be observed even in the same model, depending e.g. on the equilibrium shape of the object being modelled.

Norbert Schappacher reviews the book "Emil Artin and Beyond-Class Field Theory and $L$-Functions" by Della Dumbaugh and Joachim Schwermer, which highlights

\section{H.-Ch. Grunau}

hans-christoph.grunau@ovgu.de

1 Institut für Analysis und Numerik, Fakultät für Mathematik, Otto-von-Guericke-Universität, Postfach 4120, 39016 Magdeburg, Germany 
the memorable moments of the development of this field. The next two books under review are concerned with geometric group theory. The first one, "Descent in Buildings", was written by Bernhard Mühlherr, Holger P. Petersson, and Richard M. Weiss, and is reviewed by Bertrand Rémy. The second one was edited by Mladen Bestvina, Michah Sageev, and Karen Vogtmann and contains ten sets of lectures notes from different fields of "Geometric Group Theory". This collection is reviewed in some detail by Ralf Köhl.

Every four years the managing editor of the Jahresbericht is elected by the members of the DMV. From 2017 on Guido Schneider (Stuttgart) will be in charge, which means that the current issue is the last one which I have to take care of. This also applies to the current editorial board. I am grateful to my coeditors Thomas Bartsch, Felix Finster, Hansjörg Geiges, Martin Grothaus, Martin Hanke, Michael Hinze, Gabriele Nebe, Guido Schneider, and Wolfgang Soergel, who have acquired a number of exciting historical and survey articles. Thomas Bartsch has been responsible mainly for the book reviews and "Classics Revisited"-section where one has often got an inspiring first impression of what is going on in special fields of mathematics. The collaboration with Springer-Verlag, in particular with Andreas Vogel, and with the VTeX-type setting company has been a great pleasure. My best wishes go to Guido Schneider for his mission as new editor-in-chief of the Jahresbericht. 